\title{
Mode Conversion at Diffracting Apertures in Millimeter and Submillimeter Wave Optical Systems
}

\author{
J. Anthony Murphy, Member, IEEE, Stafford Withington, Member, IEEE, and Aidan Egan
}

\begin{abstract}
Diffraction effects, which occur when the fields in a beam waveguide are truncated at absorbing stops, can be conveniently analyzed using scattering-matrix theory applied to Gaussian-beam modes. We present recursion relationships for the elements of the scattering matrix when a nonaxially-symmetric beam illuminates an axially symmetric stop. We demonstrate the technique by calculating the total loss and beam profiles in a system comprising a diagonal horn, a lens, a window, and two off-axis mirrors. The finite size of each component is taken into account.
\end{abstract}

\section{INTRODUCTION}

$\mathbf{I}^{N}$ $\mathrm{N}$ a recent paper we described a technique for determining he power that is lost when the beam in a beam waveguide is truncated by an axially symmetric stop [1] [2]. The technique is based on the principle that for a Gaussian-mode system, with a given set of mode coefficients, the scale size and form of the beam at a plane are completely characterized by the Gaussian radius and phase slippage respectively. Hence, the power lost at a circular aperture is fully determined by two quantities both of which are easily calculated using single-mode design techniques [3].

If a beam waveguide has a number of truncating components, the beam profile at the exit pupil and the total loss depend on the degree to which beam diffracts at each of the apertures. Consequently, although the technique described in [2] is invaluable for determining how big a component has to be in order to avoid truncation, it only gives a limited amount of information about the way in which a system behaves when a significant amount of truncation occurs. In this paper, we describe a numerically-efficient technique for calculating the beam profiles and loss at any plane in a complicated quasioptical system for which diffraction losses cannot be ignored. The scheme provides an excellent way of checking in detail the performance of systems designed using the above "single-mode" technique.

The presence of an absorbing stop in a beam waveguide causes power to be scattered between modes [4]. That is to say, if we represent the incident beam by a vector whose compo-

Manuscript received September 14, 1992; revised February 27, 1993.

J.A. Murphy is with the Experimental Physics Department, Maynooth College, Co. Kildare, Ireland.

S. Withington is with Cavendish Laboratory, Madingley Road, Cambridge CB3 OHE, England.

A. Egan is with the Department of Pure and Applied Physics, Trinity College, Dublin 2, Ireland.

IEEE Log Number 9211927. nents are the coefficients of a Gaussian-mode expansion, then we can truncate the beam by linearly operating on the vector to produce a new vector which describes the transmitted field. For this technique to be useful, it must be possible to calculate the elements of the scattering matrix in a numerically efficient manner. In this paper, we present recursion relationships for the elements of the scattering matrix when a non-axiallysymmetric beam illuminates an axially-symmetric stop. We illustrate the technique by analyzing a system comprising a diagonal horn, a lens, a window and two off-axis mirrors. The finite size of each component is taken into account.

\section{THEORY}

For a circular stop that is coaxial with the direction of propagation and perfectly absorbing outside the transmitting region, the propagating fields are most conveniently described as a sum of Associated Laguerre-Gaussian modes $E(r, \theta, z)=$ $\Sigma_{n, \alpha} A_{n \alpha}^{c} \psi_{n}^{\alpha, \cos }(r, \theta, z)+A_{n \alpha}^{s} \psi_{n}^{\alpha, \sin }(r, \theta, z)$, where the modes are defined by

$$
\begin{aligned}
\psi_{n}^{\alpha, \cos / \sin }(r, \theta, z)= & \sqrt{\frac{2\left(2-\delta_{0 n}\right) n !}{\pi W^{2}(n+\alpha) !}}\left[2\left(\frac{r}{W}\right)^{2}\right]^{\frac{\alpha}{2}} \\
& \cdot L_{n}^{\alpha}\left[2\left(\frac{r}{W}\right)^{2}\right] \exp \left[-\left(\frac{r}{W}\right)^{2}\right] \\
& \cdot \exp \left[-j k\left(z+\frac{r^{2}}{2 R}\right)+j(2 n+\alpha+1) \phi_{0}\right] \\
& \cdot\left\{\begin{array}{l}
\cos (\alpha \theta) \\
\sin (\alpha \theta)
\end{array}\right\}
\end{aligned}
$$

The associated Laguerre polynomials are defined as in [5], and $W, R$, and $\phi_{0}$ have their usual significance [6]. If the circular aperture, at $z=z_{o}$, has radius $a$ then the field at the aperture has the form $E_{a p}\left(r, \theta, z_{o}\right)=0$ for $r>a$, and we can write

$$
\begin{aligned}
E_{a p}\left(r, \theta, z_{o}\right)= & \sum_{n, \alpha} A_{n \alpha}^{c} \psi_{n}^{\alpha, \cos }\left(r, \theta, z_{o}\right)^{T} \\
& +\sum_{n, \alpha} A_{n \alpha}^{S} \psi_{n}^{\alpha, \sin }\left(r, \theta, z_{o}\right)^{T},
\end{aligned}
$$

where $T$ denotes a truncated mode. Since a truncated mode is not a true mode of propagation, some of the power in a given incident mode will be redistributed between the other modes. Mathematically, we can write each truncated mode as a sum 
of true propagating modes:

$$
\begin{aligned}
& \psi_{n}^{\alpha, \cos }\left(r, \theta, z_{o}\right)^{T}=\sum_{m, \alpha^{\prime}} S_{m \alpha^{\prime}, n \alpha}^{c} \psi_{m}^{\alpha^{\prime}, \cos }\left(r, \theta, z_{o}\right) \\
& \text { and } \psi_{n}^{\alpha, \sin }\left(r, \theta, z_{o}\right)^{T}=\sum_{m, \alpha^{\prime}} S_{m \alpha^{\prime}, n \alpha}^{c} \psi_{m}^{\alpha^{\prime}, \sin }\left(r, \theta, z_{o}\right) .
\end{aligned}
$$

Because of the symmetry of the aperture, $S_{m \alpha^{\prime}, n \alpha}^{c}$ and $S_{m \alpha^{\prime}, n \alpha}^{s}$ are given by

$$
S_{m \alpha^{\prime}, n \alpha}^{c}=S_{m \alpha^{\prime}, n \alpha}^{s}=\delta_{\alpha \alpha^{\prime}} I_{m, n}^{\alpha} \exp \left[2(n-m) j \phi_{o}\right],
$$

where

$$
I_{m, n}^{\alpha}\left(x_{t}\right)=\int_{0}^{x_{t}} \frac{x^{\alpha} L_{m}^{\alpha}(x) L_{n}^{\alpha}(x) e^{-x}}{\sqrt{(m+\alpha) !(n+\alpha) ! / m ! n !}} d x .
$$

and $x_{t}=2(a / W)^{2}$. The field can then be re-expressed in terms of the propagating modes:

$$
\begin{aligned}
E_{a p}\left(r, \theta, z_{o}\right)= & \sum_{m, \alpha^{\prime}} B_{m \alpha^{\prime}}^{c} \psi_{m}^{\alpha^{\prime}, \cos }\left(r, \theta, z_{o}\right) \\
& +B_{m \alpha^{\prime}}^{s} \psi_{m}^{\alpha^{\prime}, \sin }\left(r, \theta, z_{o}\right)
\end{aligned}
$$

where $B_{m \alpha^{\prime}}^{c / s}=\sum_{n, \alpha} S_{m \alpha^{\prime}, n \alpha}^{c / s} A_{n \alpha}^{c / s}$. We can regard $S_{m \alpha^{\prime}, n \alpha}^{c / s}$ as a scattering matrix, which operates on the vector $A_{n \alpha}^{c / s}$ of incident mode coefficients to yield the vector $B_{m \alpha^{\prime}}^{c / s}$, of transmitted mode coefficients. Thereafter, the beam propagates with the new set of mode amplitudes until the next aperture is encountered.

An important feature of the technique is that it is possible to derive recursion relationships for the overlap integrals $I_{m, n}^{\alpha}\left(x_{t}\right)$, and these enable the truncation calculations to be evaluated analytically. This allows the scattering matrix to be determined quickly without the need for a whole series of lengthy numerical integrations. The relationships (for $\alpha \geq 0$ ) are

$$
\begin{aligned}
I_{m+1, n+1}^{0}= & I_{m, n}^{0}+\left(L_{m}\left(x_{t}\right)-L_{m+1}\left(x_{t}\right)\right) \\
& \cdot\left(L_{n+1}\left(x_{t}\right)-L_{n}\left(x_{t}\right)\right) e^{-x_{t}} \\
\sqrt{\alpha+1} I_{m, 0}^{\alpha+1}= & \sqrt{m+\alpha+1} I_{m, 0}^{\alpha} \\
& -\sqrt{m+1} I_{m+1,0}^{\alpha} \quad \text { for } m>0 \\
\sqrt{n+\alpha+1} I_{m, n}^{\alpha+1}= & \sqrt{n} I_{m, n-1}^{\alpha+1} \\
& +\sqrt{m+\alpha+1} I_{m, n}^{\alpha} \\
& -\sqrt{m+1} I_{m+1, n}^{\alpha} \\
& \text { for } m, n>0 .
\end{aligned}
$$

To initiate the above series we need to compute $I_{n, 0}^{0}\left(=I_{0, n}^{0}\right)$, and these can be determined by using

$$
\begin{aligned}
& I_{0,0}^{0}=1-\exp \left[-x_{t}\right] \\
& I_{0, n}^{0}=\left(L_{n-1}\left(x_{t}\right)-L_{n}\left(x_{t}\right)\right) \exp \left[-x_{t}\right] .
\end{aligned}
$$

Note, that if we wish to truncate the series at $I_{m_{\max }, n_{\max }}^{\alpha_{\max }}$ then the last of the recursion relationships above requires determining $I_{m, n}^{0}$ up to $I_{m_{\max }+\alpha_{\max }, n}^{0}, I_{m, n}^{1}$ up to $I_{m_{\max }+\alpha_{\max }-1, n}^{1}$ and $I_{m, n}^{2}$ up to $I_{m_{\max }+\alpha_{\max }-2, n}^{2}$, etc.
TABLE I

Beam Parameters of an Optical System Comprising a Diagonal Horn, a Lens and Two Off-Axis Mirrors. The Beam Parameters are Calculated at $400 \mathrm{GHz}$ The Horn is $19.0 \mathrm{~mm}$ LONG AND HAS A $3.5 \mathrm{~mm}$ SoUARE APERTURE.

\begin{tabular}{||l|c|c|c|c|c|c||}
\hline \hline component & separation(mm) & $W(\mathrm{~mm})$ & $a / W$ & $\Delta \phi($ deg.) & $P_{\mathrm{co}}(\%)$ & Loss (\%) \\
\hline & & & & & & \\
\hline & & 1.35 & & -26 & & \\
\hline virtual waist & 3.8 & & & & & \\
\hline horn aperture & & 1.5 & & 0 & 100.0 & \\
\hline & 32 & & & & & \\
\hline lens (f=32) & & 6.5 & 3.8 & 52 & 98.3 & 1.8 \\
\hline window (50mm) & 86 & & & & & \\
\hline & 280 & 5.1 & 4.9 & 90 & 98.1 & 1.8 \\
\hline mirror $(\mathrm{f}=280)$ & & 14.1 & 2.5 & -21 & 97.9 & 1.6 \\
\hline & 280 & & & & & \\
\hline image & & 13.2 & & 0 & & \\
\hline & 350 & & & & & \\
\hline mirror (f=350) & & 14.7 & 2.4 & 25 & 97.6 & 1.9 \\
\hline & 350 & & & & & \\
\hline cass focus & & 6.3 & & 90 & & \\
\hline \hline
\end{tabular}

\section{EXAMPLE}

As an example, consider the $200-900 \mathrm{GHz}$ optical system listed in Table I [1], where a diagonal horn is coupled to a submillimetre-wave telescope through a lens and two off-axis mirrors. The horn is part of a superconducting mixer which is located in a cryostat, and therefore the beam has to pass through a window which must be made as small as possible. In Table I, we list the normalized truncation radius $(a / W)$, the phase slippage from the aperture of the horn, and the fractional amount of power remaining in the co-polar beam $P_{c o}$. Since the total amount of co-polar power contained in the beam is proportional to $\int\left|E_{c o}\right|^{2} d A$, where the integral is over the cross sectional area of the beam, this implies that:

$$
\begin{aligned}
P_{c o} & =\frac{\int_{\text {comp }}\left|E_{c o}\right|^{2} d A}{\int_{\text {horn }}\left|E_{c o}\right|^{2} d A} \\
& =\frac{\sum_{n, \alpha}\left|B_{n, \alpha}^{c}\right|^{2}+\left|B_{n, \alpha}^{s}\right|^{2}}{\sum_{n, \alpha}\left|A_{n, \alpha}^{c}\right|^{2}+\left|A_{n, \alpha}^{s}\right|^{2}} .
\end{aligned}
$$

The $A_{n, \alpha}^{c / s}$ are the mode coefficients for the co-polar field at the horn aperture, and the $B_{n, \alpha}^{c / s}$ are the resulting transmitted mode coefficients after scattering at each stop where truncation occurs in the beam guide, up to and including the relevant component. If we use the technique described in [2] to calculate the amount of power lost at an individual aperture (and assuming no other truncation loss in the optical system) we arrive at the figures given in the last column. In Fig. 1, we use the mode coefficients, phase slippages, and Gaussian radii to reconstruct the beam profiles at a number of different planes.

The last column is the loss at each component if one assumes that the beam remains unchanged after passing through each stop up to that point. The fifth column, however, shows that the total loss is, in reality, much less than the sum of these individual losses. Clearly, in this particular case, the apertures are many wavelengths in diameter, and the beam diffracts only slightly after passing through each stop. Consequently, the first lens after the mixer truncates the beam, and this truncated beam passes all of the way through the optical system without much further interference. From a modal point of view, the 


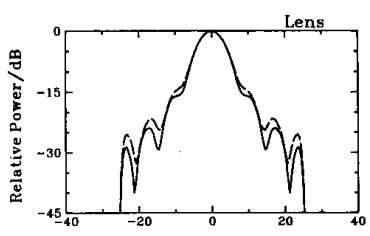

Distance off axis $/ \mathrm{mm}$

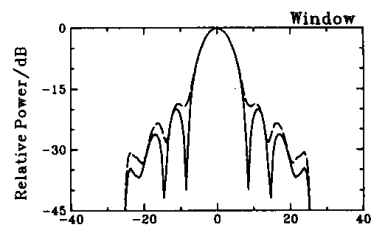

Distance off axis $/ \mathrm{mm}$

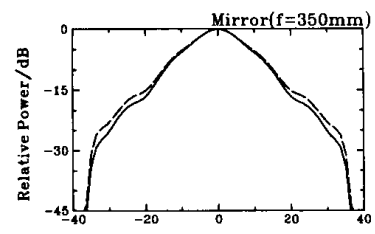

Distance off axis $/ \mathrm{mm}$

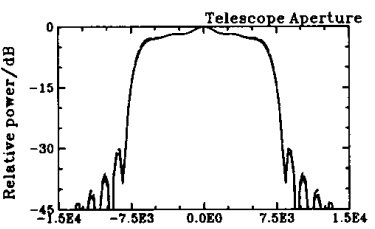

Distance off axis $/ \mathrm{mm}$

(a)

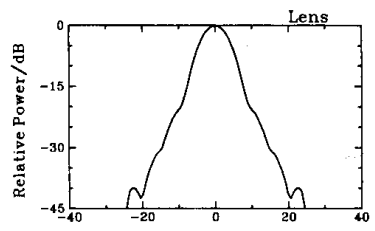

Distance off axis $/ \mathrm{mm}$

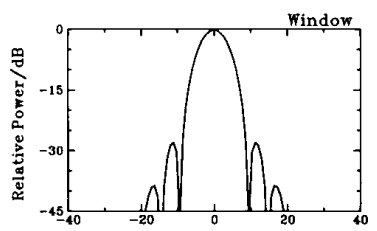

Distance off axis $/ \mathrm{mm}$
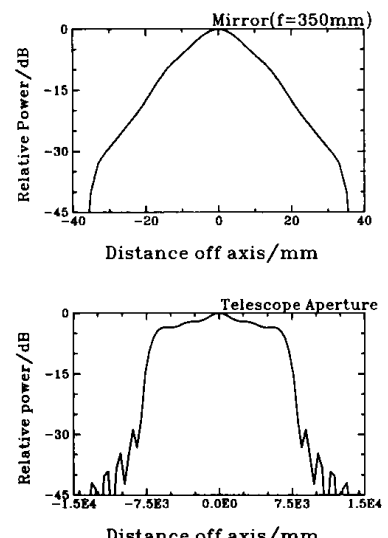

(b)

Fig. 1. (a) Reconstructed beam profiles at various planes in the example optical system fed by a diagonal horn (see Table I) in the diagonal 45 deg or X-Y directions. The two curves correspond to the copolar power (solid line) and total power (dashed line). Also shown is the beam at the aperture of a $15 \mathrm{~m}$ telescope to which the system is coupled. (b) Reconstructed beam profiles at various planes in the example optical system is the vertical and horizontal (V-H) directions. The diagonal horn does not have any cross-pola components in the $\mathrm{V}-\mathrm{H}$ direction, hence the copolar and total powers are equal. Also shown in the beam at the aperture of a $15 \mathrm{~m}$ telescope to which the system is coupled.

first lens is acting as a mode filter which rejects some of the higher order modes. In low noise receivers it is, of course, desirable to reject the high-order modes at low temperatures because then less noise is coupled into the system.

\section{ACKNOWLEDGMENT}

The authors would like to thank EOLAS and the British Council for financing some of the travel costs incurred.

\section{REFERENCES}

[1] S. Withington, J. A. Murphy, A. Egan, and R. E. Hillis, "A broadband quasioptical system for submillimetre wave radio-astronomy receivers," Int. J. Infrared and Millimeter Waves, vol. 13, pp. 1515-1537, 1992.

[2] J. A. Murphy, A. Egan, and S. Withington, "Truncation in millimetre and submillimetre-wave optical systems," submitted to IEEE Trans. Antennas Propagat.

[3] P.F. Goldsmith, "Quasi-optical techniques at millimeter and submillimeter wavelengths," in Infrared and Millimeter Waves, vol. 8, no. 9, pp. 277-343, 1982.

[4] R. Padman, and J.A. Murphy, "A scattering matrix formulation for Gaussian beam mode analysis," in Proc. IEE/URSI 7th Int. Conf. on Antennas and Propagation, ICAP, York, England, Apr. 1991, pp. 201-204.

[5] I. S. Gradshteyn and I. M. Ryzhik, Table of Integrals, Series and Products. London: Academic Press, 1980.

[6] H. Kogelnik and T. Li, "Laser beams and resonators," Proc. IEEE vol. 54, pp. 1312-1329, 1966.

J. Anthony Murphy (M'88) was born in Cork, Ireland on October 30, 1954. He received the B.Sc. and M.Sc. degrees in Experimental Physics from University College, Cork, Ireland, in 1977 and 1979, respectively, the M.S. degree in Physics from the California Institute of Technology in 1981 and the Ph.D. degree in Physics from the University of Cambridge, England, in 1986.

From 1985 to 1987 he was a Research Associate at the Cavendish Laboratory, Cambridge, where he was engaged in receiver development for the James Clerk Maxwell Telescope on Mauna Kea, Hawaii. In 1988 he was appointed as lecturer in the Experimental Physics Department, St. Patrick's College, Maynooth, Ireland. His main research interest is in millimeter- and submillimeter-wave optics.

Stafford Withington (M'89) was born in Chesterfield, England, on May 27, 1957. He received the B. Eng. Hons. degree, first class, from the University of Bradford, England, in 1979, and the Ph.D. degree in Radio Astronomy from the University of Manchester, England in 1983

He has held a number of positions, including a lectureship in the Department of Electrical Engineering at Sheffield University and a Royal Society Overseas Fellowship in the Department of Radio and Space Science at Chalmers University of Technology, Sweden. He is currently a Senior Research Associate in the Department of Physics at Cambridge University and a Fellow of Downing College, Cambridge. In recent years he has concentrated on the development of low-noise submillimeter-wave receivers for use in radio astronomy, and he is particularly interested in superconducting detector technology and submillimeter-wave optics.

Aidan Egan was born in Offaly, Ireland, on April 30, 1969. He received the B.Sc. and M.Sc. degrees in Physics from St. Patrick's College, Maynooth, Ireland, in 1990 and 1992 respectively. Currently he is pursuing the Ph.D. degree in Physics at Trinity College, Dublin, Ireland, where his research interests are in modelling of multi-electrode semiconductor lasers, selfpulsation and optical synchronization. 